\title{
DIAGNOSTIC ACCURACY OF DOPPLER SONOGRAPHY AND MAGNETIC RESONANCE VENOGRAPHY FOR THE ASSESSMENT OF IMPAIRED OUTFLOW FROM THE INTERNAL JUGULAR VEIN IN PATIENTS WITH MULTIPLE SCLEROSIS
}

\author{
Marcin Hartel', Ewa Kluczewska², Marian Simka ${ }^{3}$ \\ 'Magnetic Resonance Imaging Department, MCD Voxel, Zabrze, Poland \\ ${ }^{2}$ Chair and Department of Radiology in Zabrze, Medical University of Silesia, Katowice, Poland \\ ${ }^{3}$ Department of Angiology, Private Healthcare Institution SANA, Pszczyna, Poland
}

\author{
ORIGINAL PAPER
}

Phlebological Review 2015; 23, 1: 20-28

DOI: $10.5114 /$ pr.2015.51628

Submitted: 08.01.2015

Accepted: 19.03.2015

\begin{abstract}
Objectives: This prospective study was aimed at evaluation of diagnostic value of Doppler sonography and magnetic resonance (MR) venography in the internal jugular vein territory.

Material and methods: There were assessed 126 patients (252 internal jugular veins). The patients were initially diagnosed using Doppler sonography and MR venography, and then catheter venography of the veins was performed. For the purpose of this study catheter venography was regarded the reference test. We interpreted sonographic findings in the context of criteria proposed by Zamboni and criteria by International Society for Neurovascular Disease (ISNVD). We applied MR protocols aimed at flow assessment in the scanned veins: fast spin echo-T2-weighted sequences with fat saturation, and axial two-dimensional time-of-flight imaging with saturation band positioned below planned slices.

Results: Catheter venography revealed lesions in 171 (67.9\%) of the veins studied, primarily stenotic jugular valves (88.0\% of abnormal veins). We found that sonographic criteria, using either Zamboni or ISNVD criteria were of poor diagnostic accuracy (Cohen's $\kappa$ coefficient $<0.1$ ). MR venography with the use of our dedicated protocols appeared to be a slightly better diagnostic tool ( $\kappa$ about 0.4 ).

Conclusions: The results of our MR imaging protocols for the assessment of abnormalities in internal jugular veins were better if compared to Doppler sonography. Yet, even MR venography was far from being perfect and at the moment neither this examination nor Doppler sonography cannot replace invasive catheter venography and should not be used as a definitive test for diagnosing anatomical and functional abnormalities in the internal jugular veins.
\end{abstract}

Key words: jugular vein, chronic cerebrospinal venous insufficiency, Doppler ultrasonography, magnetic resonance venography.

\author{
ADDRESS FOR CORRESPONDENCE \\ Marian Simka \\ Department of Angiology \\ Private Healthcare Institution SANA \\ Pszczyna, Poland \\ e-mail: mariansimka@poczta.onet.pl \\ email: mariansimka@poczta.onet.pl
}

\section{INTRODUCTION}

Chronic cerebrospinal venous insufficiency (CCSVI) was initially defined as a clinical syndrome comprising stenoses of the internal jugular and/or azygos veins, characterised by collateral venous outflow and reduced cerebral blood flow $[1,2]$. Contrary to other venous pathologies characterised by prevailing post-thrombotic abnormalities, the majority of CCSVI patients present with valvular and functional anomalies. Such a unique vascular pathology is not often seen in the venous system, and for the time being no established non-invasive method for the assessment of such a condition exists [3]. Although in most of the published studies a diagnosis of CCSVI was given using Doppler sonography or MR venography, research on the validity of these tests was done primarily in the context of potential association of CCSVI with multiple sclerosis (MS) [1, 4-7]. However, it seems more accurate to validate a test against the established gold standard, and not against the presence or absence of the disease [8]. In addition, CCSVI - defined as an anomalous outflow from the brain and spinal cord is probably not a single clinical entity, but rather a group of different anatomical and functional abnormalities that in some individuals can even come together. Patients can present with diminished inflow to the internal jugular vein (IJV) resulting from decreased cerebral venous flow, with the IJV externally compressed (usually by aberrant 
adjacent muscles, arteries, or bones), with hypoplastic IJV, or with stenotic jugular valves incurring flow retention [3]. This heterogeneity of CCSVI is probably responsible for the low diagnostic reliability of non-invasive tests. Also, considering these many faces of CCSVI, it is easier to understand why the results of studies on the prevalence of this clinical entity are so discordant. Not only were the authors using different diagnostic modalities and protocols, but probably they were also interpreting the findings of the tests differently and looking for different irregularities [3].

Our prospective study was aimed at the evaluation of diagnostic values of the above-mentioned non-invasive modalities, using catheter venography as the reference test.

\section{MATERIAL AND METHODS}

This survey was aimed at the assessment of the diagnostic efficacy of Doppler sonography and MR venography in the IJV territory. In this study 126 patients with clinically defined MS were prospectively assessed. There were 87 women and 39 men, and the patients were aged 22-69 years, with a median age of 50 years. The patients were initially examined using Doppler sonography and MR venography. Then, they underwent standard catheter venography of their IJVs and the azygous vein. For the purpose of this study, catheter venography was regarded as the reference test, and the results of this examination were compared to those of Doppler sonography and MR venography. Patients positive for CCSVI in catheter venography then underwent venous angioplasty of the stenosed veins. Doppler sonography and catheter venography were performed in all 126 patients, whereas MR venography was done only in 82 patients. The azygous vein lesions that were detected using either catheter or MR venography are not discussed in this paper.

This study was approved by the Bioethical Committee of the Regional Silesian Board of Physicians in Katowice, Poland: approval No: 20/2011, and the study was registered at ClinicalTrials.gov - identifier: NCT01425554. All patients gave written consent to undergo the procedures and diagnostic tests.

\section{Catheter venography}

The details and interpretation of catheter venography used in this study have been described elsewhere [8-10]. The following venographic flow patterns in the internal jugular veins were regarded as abnormal:

- no outflow of contrast through the vein,

- venous outflow slowed down, i.e. a retention of injected contrast in the examined vein longer that one cardiac cycle,

- reversed flow direction (reflux),

- outflow through collaterals,

- prestenotic dilation of the vein associated with slower flow or reflux,
- complete occlusion or agenesia of the vein,

- intraluminal structures (webs, septa, or membranes), hypoplasia, or narrowing of the vein compromising outflow, i.e. incurring the retention of injected contrast, reflux or collateral outflow,

- jugular valve (the valve located just above the junction of the IJV with the brachiocephalic vein) was recognised as pathological if such a valve compromised the outflow in a similar way as other intraluminal structures (Fig. 1).

\section{Doppler sonography}

Imaging and assessment of the IJVs using colour Doppler sonography was conducted with an GE LOGIQ-e ultrasound machine with an $8-\mathrm{MHz}$ linear probe (GE Healthcare, Cleveland, Ohio, USA) [8]. The probe applied minimal pressure to the skin to prevent undesired compression of the examined veins: the IJVs and vertebral veins (VVs). We did not examine intracranial veins. Since all patients assessed suffered from MS, the sonographer was not blinded regarding the clinical diagnosis of the patients. Nonetheless, he did not know the results of the $\mathrm{MR}$ and catheter venographies (the latter was performed after the two non-invasive tests). Examinations were performed with patients in the supine and sitting positions. IJVs were evaluated in three locations:

- in the valve area (junction with the brachiocephalic vein $)-(J 1)$,

- in the middle part of the vein - (J2),

- in the upper part of the vein (cranially from its junction with the facial vein) - (J3).

The following parameters were evaluated in each of these locations and repeated in the supine and sitting positions:

- flow direction (towards heart, reversed, bidirectional),

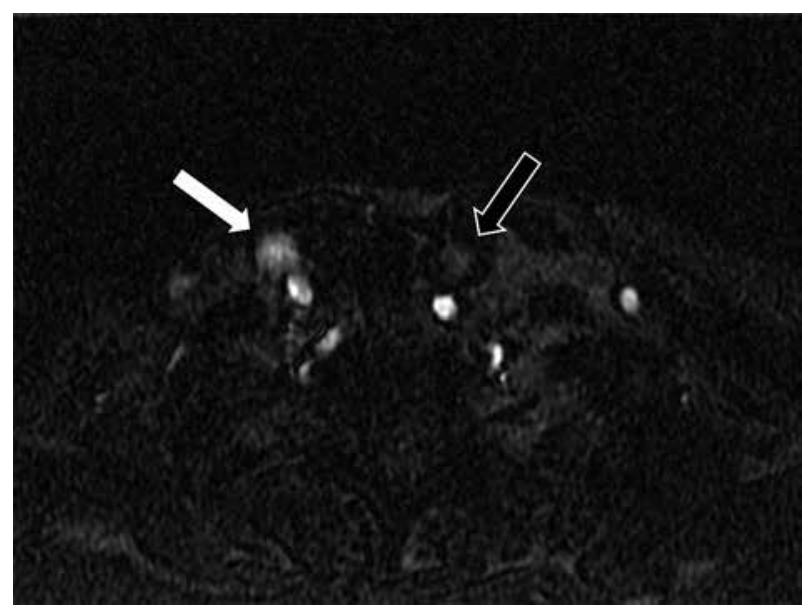

Fig. 1. Catheter venography of the right internal jugular vein. Severe stenosis (arrow) at the level of the jugular valve incurring prestenotic dilatation of the vein and outflow of injected contrast through collaterals 
- presence of reflux (flow towards the brain) longer than 0.8 seconds,

- absence of flow,

- peak flow velocity,

- presence of intraluminal defects (valve, septum, membrane),

- cross-sectional area (CSA).

Vertebral veins were assessed in the middle part of the neck, in the best visible segment. VVs, similarly to the IJVs, were examined in the supine and sitting positions, and the same parameters - except for the presence of intraluminal defects and CSA - were evaluated. We interpreted our findings in the context of two sets of sonographic criteria: proposed by Zamboni (but not using the evaluation of the intracranial veins) and criteria suggested by an expert panel of the International Society for Neurovascular Disease (ISNVD).

Sonographic criteria of CCSVI by Zamboni:

1. Reflux. Constant reflux (>0.8 s) in a single IJV or VV, in sitting or supine position.

2. Stenosis/B-mode anomalies. Reduction of the CSA of IJV less than $0.3 \mathrm{~cm}^{2}$ in both body positions, and the presence of an intraluminal defect (such as webs, septa, or malformed valves).

3. No flow. Absence of Doppler signal in IJV or VVs in both supine and sitting positions.

4. Negative $\triangle C S A$. CSA of the IJV, which was greater in the sitting position than in the lying position, or appeared unchanged despite change in posture [11]. ISNVD Sonographic criteria of CCSVI:

1. Reflux. Bidirectional flow in the IJV in both postures, or bidirectional flow in one position with absence of flow in the other position.

2. Stenosis/B-mode anomalies. Reduction of the CSA of IJV less than $0.3 \mathrm{~cm}^{2}$ in the supine position, or the presence of an intraluminal defect combined with haemodynamic changes (increased velocity, absence of flow, reverse flow, etc.).

3. No flow. Absence of Doppler signal in IJV or VVs in both sitting and upright positions, or in one posture but with bidirectional flow detected in the other position.

4. Negative $\triangle C S A$. CSA of the IJV, which was greater in the sitting position than in the lying position, or appeared unchanged despite change in posture [12].

\section{Interpretation of sonographic findings}

We regarded a criterion to be positive if the required parameters were met in at least one segment of the IJV: $\mathrm{J} 1, \mathrm{~J} 2$, or J3. We interpreted as a reflux (Zamboni's criterion 1) only completely reversed flow detected in the entire cross-section of the vein. Of note, we did not interpret as a reflux vortical flow or artefacts produced by whirls in the proximity to valve cusps. Also, we interpreted as intraluminal defects (Zamboni's criterion 2) all sonographically detectable valves, since it was very difficult to set a reasonable threshold between 'normal' and 'malformed' valves. Similarly, since it was not possible to set such a threshold between 'normal' and 'increased' flow velocities (ISNVD criterion 2), we interpreted as a stenosis the presence of intraluminal defects combined with absent or reversed flow [8]. Final interpretation was done by an experienced sonographer, and the vein was interpreted as abnormal if there were:

- at least one positive Zamboni's criterion,

- at least two positive Zamboni's criteria,

- at least one positive ISNVD criterion,

- at least two positive ISNVD criteria (Table 1).

In addition to Zamboni's and ISNVD CCSVI sonographic diagnostic criteria, we also evaluated the following sonographic parameters in the IJVs:

- flow direction (towards heart, reversed, bidirectional),

- presence of reflux (flow towards brain) longer than 0.8 seconds,

- absence of flow,

- value of peak flow velocity,

- presence of intraluminal defects (valve, septum, membrane),

- CSA,

- quotient of maximal and minimal cross-sectional diameter,

- difference of cross-sectional areas in the sitting versus upright positions $(\triangle \mathrm{CSA})$.

Each of these parameters was measured at three different locations: at J1, J2, and J3 levels, and also (except for $\triangle \mathrm{CSA}$ ) in the sitting and upright body positions, thus in total 45 different parameters were evaluated. An association of these sonographic parameters with venographically-detected abnormalities was further tested statistically using multivariate regression analysis.

\section{Magnetic resonance venography}

Magnetic resonance venographies were acquired using a GE 1.5 Tesla HDx magnetic resonance scanner equipped with an 8-channel head coil (GE Medical Systems, Milwaukee, Wisconsin, USA). For every subject the head was maintained straight. Fast scout gradient-echo images were acquired initially in the coronal, sagittal, and transverse planes for localisation. Then the following sentences were acquired:

- two-dimensional fast imaging employing steady-state acquisition (2D FIESTA) in three planes: axial, coronal, and sagittal (slice thickness/TR/TE/NEX $=4 \mathrm{~mm} /$ 4.03/1.7/1),

- axial fast spin echo-T2-weighted sequences with fat saturation (FSE+FatSat), using the following parameters: FatSat, slice thickness/TR/TE/NEX $=4 \mathrm{~mm} / 3440 / 105.9 / 2$,

- axial two-dimensional time-of-flight (2D TOF) imaging with a saturation band positioned below planned slices (slice thickness/TR/TE/NEX $=3 \mathrm{~mm} / 9.1 / 3.7 / 1$ ) [13]. 
Table 1. Diagnostic values of sonographic and MR venographic CCSVI criteria, including likelihood ratios according to the Bayes' theorem and the Cohen's $\kappa$ coefficient

\begin{tabular}{|c|c|c|c|c|c|}
\hline & Sensitivity (\%) & Specificity (\%) & $\begin{array}{l}\text { Positive and negative } \\
\text { predictive values (\%) }\end{array}$ & $\begin{array}{l}\text { Bayesian likelihood } \\
\text { ratios of a positive and } \\
\text { negative result (\%) }\end{array}$ & $\begin{array}{c}\text { Cohen's } \kappa \\
\text { coefficient (\%) }\end{array}$ \\
\hline \multicolumn{6}{|c|}{ Diagnostic accuracy of Doppler sonography (252 veins studied) } \\
\hline $\begin{array}{l}\text { at least one positive } \\
\text { Zamboni's criterion }\end{array}$ & 92.3 & 12.5 & $70.6 / 41.7$ & $69.0 / 43.4$ & 0.062 \\
\hline $\begin{array}{l}\text { at least two positive } \\
\text { Zamboni's criteria }\end{array}$ & 27.2 & 79.7 & $74.6 / 33.3$ & $73.9 / 34.1$ & 0.047 \\
\hline $\begin{array}{l}\text { at least one positive } \\
\text { ISNVD criterion }\end{array}$ & 91.2 & 12.5 & $70.3 / 38.5$ & $68.8 / 40.2$ & 0.047 \\
\hline $\begin{array}{l}\text { at least two positive } \\
\text { ISNVD criteria }\end{array}$ & 45.3 & 66.3 & $74.3 / 36.1$ & $73.9 / 36.4$ & 0.094 \\
\hline \multicolumn{6}{|c|}{ Diagnostic accuracy of MR venography (164 veins studied) } \\
\hline $\begin{array}{l}\text { abnormal FatSat MR } \\
\text { venography }\end{array}$ & 93.2 & 37.0 & $79.1 / 68.0$ & 75.8/72.1 & 0.400 \\
\hline $\begin{array}{l}\text { abnormal 2D TOF MR } \\
\text { venography }\end{array}$ & 89.8 & 43.5 & $80.3 / 62.5$ & $77.1 / 66.8$ & 0.407 \\
\hline $\begin{array}{l}\text { abnormal FatSat or 2D } \\
\text { TOF MR venography }\end{array}$ & 93.2 & 34.8 & $78.6 / 66.7$ & $75.2 / 70.9$ & 0.378 \\
\hline
\end{tabular}

The first imaging technique was used primarily for the evaluation of venous anatomy, whereas the two others provided information on venous flow characteristics. In this survey we analysed the results of FSE+FatSat and 2D TOF imaging. There were also some infrequent cases of severe narrowing of the IJVs demonstrated with 2D FIESTA imaging, but such abnormal results of this imaging technique were not further analysed statistically.

In this study we interpreted the MR venography of the internal jugular veins as pathological if the vein was found abnormal in:

- FSE+FatSat MR venography,

- 2D TOF MR venography,

- at least in one of the above-mentioned diagnostic modalities.

Such an interpretation was done by an experienced neuroradiologist, and the vein was interpreted as abnormal if there were features of slowed or reversed flow. In the case of the FSE+FatSat MR venography it meant the presence of bright signals inside the vein (Figs. 2 and 3). The $2 \mathrm{D}$ TOF MR venography was interpreted as abnormal if the signal was blurred and diminished (in severe cases there were even black spots inside the vein) (Figs. 4 and 5). The neuroradiologist knew that all patients suffered from MS, but he did not know the results of Doppler sonography and catheter venography.

\section{Statistical analysis}

We evaluated the diagnostic accuracy (sensitivity, specificity, positive and negative predictive values, and the Cohen's $\kappa$ coefficient) of non-invasive tests: Doppler sonography and MR venography using results of catheter venography as the reference test. Since in the studied group the prevalence of venographic abnormalities of the IJVs was very high, in addition to classical statistics we

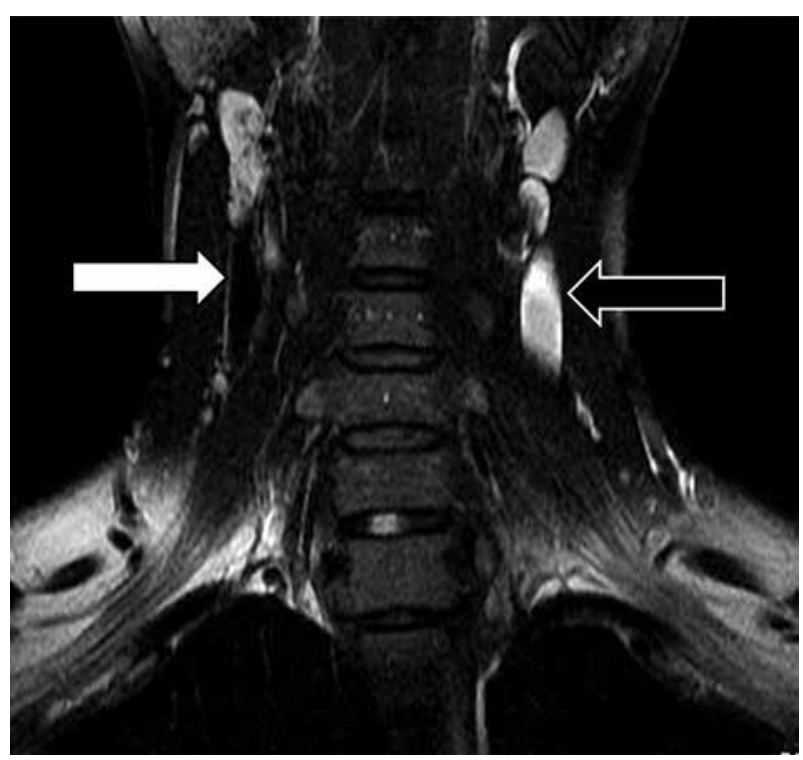

Fig. 2. Coronal FSE+FatSat MR venography of the internal jugular veins. Normal flow in the right internal jugular vein (white arrow) demonstrated by low intensity of the signal inside this blood vessel (the veins are dark); the left internal jugular vein (black arrow) is whitish, which is suggestive of venous blood stasis 


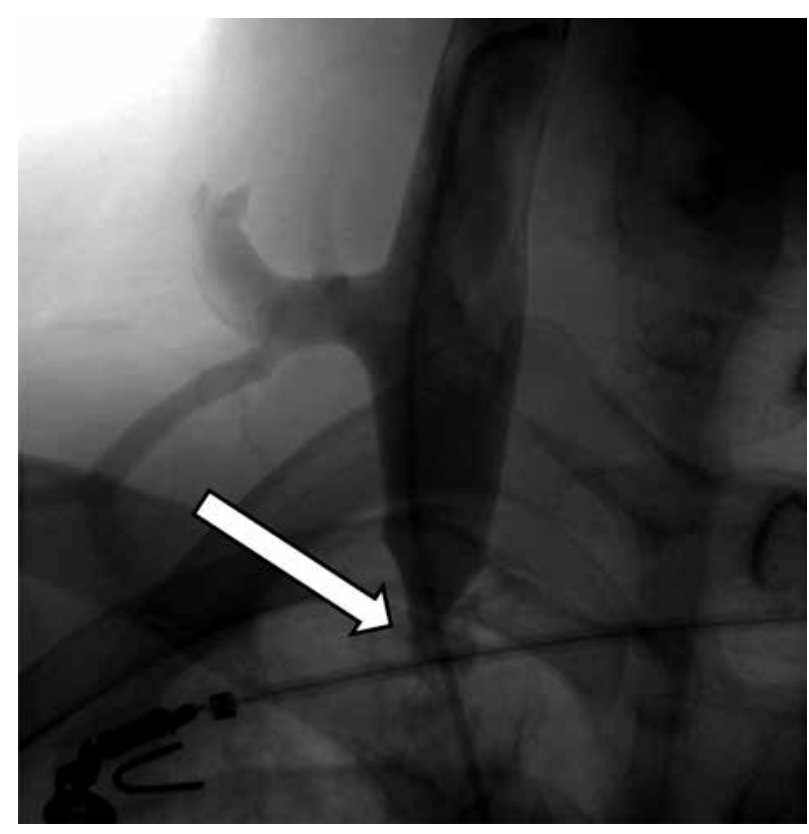

Fig. 3. A similar coronal FSE+FatSat MR venography of another patient with impaired outflow from the left internal jugular vein

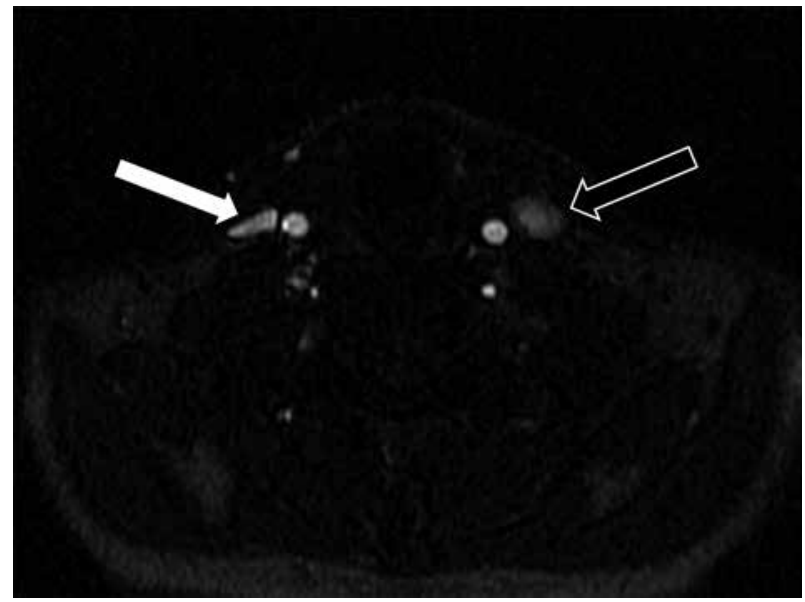

Fig. 5. A similar axial 2D TOF MR venography of another patient with impaired outflow from the left internal jugular vein

also applied the Bayesian approach and calculated the likelihood ratios of positive and negative results, assuming that prior probability of an abnormality in the IJV was $67.9 \%$ (equal to the prevalence of venographically-demonstrable lesions in this group of MS patients).

We also performed multivariate regression analysis, testing the null hypothesis that the above-mentioned 45 sonographic parameters were significantly associated with abnormalities revealed by means of catheter venography. For this purpose we used the one-way analysis of Jaccard's similarities (ANOSIM), applying the squared Mahalanobis' and the Gower's distance measures. Significance of $p$ values was set at $p<0.05$. This part of the statistical analysis was done using the PAST data analysis package (version 2.09; University of Oslo, Norway).

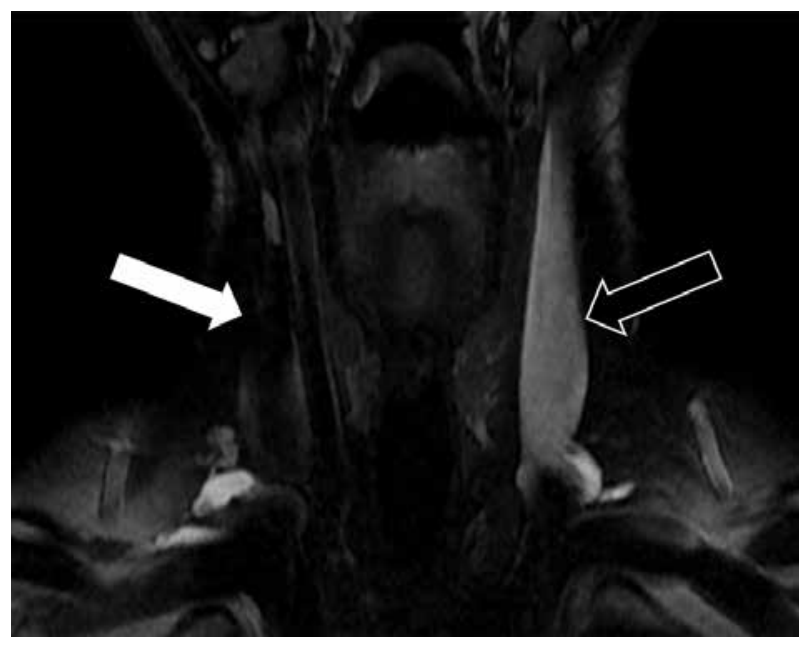

Fig. 4. Axial 2D TOF MR venography of the internal jugular veins just above the jugular valves. Normal flow in the right internal jugular vein (white arrow) demonstrated by bright signal inside this blood vessel; conversely, the left internal jugular vein (black arrow) presents with minimal signal intensity, thus suggesting venous outflow has slowed

\section{RESULTS}

\section{Safety profile of catheter venography}

There were no serious adverse events associated with catheter venography, such as: bleeding requiring blood transfusion, thromboembolism, injury to arteries or nerves, or cardiac tachyarrhythmias in this studied group of MS patients.

\section{Prevalence of venographic abnormalities in the internal jugular veins}

Catheter venography revealed anatomical and haemodynamic abnormalities meeting the above-described definitions of abnormal venographic pattern in 171 $(67.9 \%)$ of the IJVs. Almost all patients, i.e. 122 of them (96.8\%), presented with at least one abnormal IJV. Stenotic lesions were found mostly at the level of jugular valves (88.0\% of abnormal veins), and less frequently we found multiple stenoses of the vein, usually including stenosis of the valve (12.8\%). There were also single cases presenting with isolated stenoses of the middle part of the IJV $(0.6 \%)$, and of the brachiocephalic vein $(0.6 \%)$.

\section{Diagnostic accuracy on non-invasive tests}

Parameters describing the diagnostic accuracy of Doppler sonography and MR venography, such as: sensitivity, specificity, positive and negative predictive values, likelihood ratios of a positive and negative result according to the Bayes' theorem, and the $\mathrm{\kappa}$ coefficient, are given in Table 1. Interestingly, in the case of MR venography, 
a better reliability of this test was revealed in the left IJV (results not shown). Nevertheless, CCSVI lesions in MS patients are more common on the left side, so this difference might just be a reflexion of this lateralisation.

\section{Results of multivariate regression analysis of sonographic parameters}

Multivariate regression analysis of the 45 sonographic parameters studied demonstrated that seven of these variables were associated with significantly higher prevalence of venographic abnormalities. These were:

- peak flow velocity at J3 in the supine position that was less than $24 \mathrm{~cm} / \mathrm{s}$,

- peak flow velocity at J2 and J3 in the sitting position that was less than $24 \mathrm{~cm} / \mathrm{s}$,

- cross-sectional area at J1 in the supine position that was less than $70 \mathrm{~mm}^{2}\left(0.7 \mathrm{~cm}^{2}\right)$,

- cross-sectional area at $\mathrm{J} 2$ and $\mathrm{J} 3$ in the sitting position that was less than $8 \mathrm{~mm}^{2}\left(0.08 \mathrm{~cm}^{2}\right)$,

- $\triangle \mathrm{CSA}$ at J1 equal to zero or negative.

However, further analysis has revealed that despite the statistical significance of these variables, there was a big overlap between venographically positive and negative patients regarding these sonographic parameters. Therefore, even these sonographic features were not very reliable in terms of diagnostic efficacy (Table 2).

\section{DISCUSSION}

A high prevalence of venous abnormalities in MS patients, as has already been demonstrated using catheter venography $[8-10,14-18]$, makes the search for a proper non-invasive test for CCSVI difficult. Since venous pathologies can be found in the majority of MS patients, theoretically any diagnostic criterion will reveal a high sensitivity and high Bayesian likelihood ratio. Importantly, in the case of highly prevalent pathology, a reliable diagnostic test should be characterised by high specificity and a Bayesian likelihood ratio of negative results that is not lower than the actual prevalence of normal findings (in the case of this study it should not be less than 32.1\%). Besides, such a reliable test should exhibit high Cohen's $\kappa$ coefficient (traditionally it is interpreted that a $\kappa$ value higher than 0.5 indicates good agreement between the tests; however, in a case of highly prevalent pathology this threshold indicating good agreement may be lower, and in this study the value of 0.4 looks reasonable).

Under the conditions of this study we found that sonographic criteria (using either Zamboni's or ISNVD criteria) for CCSVI were of poor diagnostic accuracy. Statistical coefficients characterising diagnostic accuracy of Doppler sonography were found to be unacceptably low. Also, Cohen's $\kappa$ coefficients of all evaluated criteria were well below 0.4 . In addition, statistical analysis of sonographic parameters not included in Zamboni's or ISNVD criteria has demonstrated that even these variables were not capable of distinguishing venographically abnormal IJVs from the normal veins. However, it is likely that actually stenotic CCSVI lesions, depending on degree of stenosis, characteristics of inflow volume, and potential for collateral outflow, can differently affect the flow at the level of such a stenosis. This may explain the discordant results of sonographic studies on CCSVI [3]. Magnetic resonance venography (using our CCSVI-dedicated protocol) appeared to be a better diagnostic tool ( $\kappa$ coefficients of about 0.4 ). Yet even this test was of limited diagnostic efficacy, and with the use of currently available imaging protocols this non-invasive test cannot replace invasive catheter venography.

There are some limitations of our study. Although the reference test for the detection of IJV abnormalities (catheter angiography) is widely regarded as the golden standard for the assessment of vascular pathologies, in the territory of jugular veins it is actually a 'tarnished' standard $[19,20]$. In CCSVI patients the most prevalent abnormality is the stenotic, 'over-competent' jugular valve [21]. Using invasive diagnostics (catheter venography) about $90 \%$ of MS patients can demonstrate such venous occlusive lesions [9, 10, 14-18]. Stenotic valves are usually seen in combination with collapsed middle and/or upper parts of the IJV. However, at the moment it remains unclear what should be interpreted as normal

Table 2. Diagnostic values of selected sonographic parameters

\begin{tabular}{lcccc}
\hline Sonographic parameter & Sensitivity (\%) & Specificity (\%) & $\begin{array}{c}\text { Positive predictive } \\
\text { value (\%) }\end{array}$ & $\begin{array}{c}\text { Negative predictive } \\
\text { value (\%) }\end{array}$ \\
\hline peak flow velocity at J3 in the supine position $<24 \mathrm{~cm} / \mathrm{s}$ & 56.4 & 76.3 & 83.6 & 44.9 \\
\hline peak flow velocity at J2 in the sitting position $<24 \mathrm{~cm} / \mathrm{s}$ & 59.9 & 53.8 & 73.6 & 38.4 \\
\hline peak flow velocity at J3 in the sitting position $<24 \mathrm{~cm} / \mathrm{s}$ & 61.2 & 45.2 & 65.0 & 41.6 \\
\hline cross-sectional area at J1 in the supine position $<70 \mathrm{~mm}^{2}$ & 55.2 & 58.8 & 74.2 & 37.9 \\
\hline cross-sectional area at J2 in the sitting position $<8 \mathrm{~mm}^{2}$ & 57.0 & 75.0 & 83.1 & 44.8 \\
\hline cross-sectional area at J3 in the sitting position $<8 \mathrm{~mm}^{2}$ & 57.6 & 63.3 & 81.1 & 43.9 \\
\hline$\Delta$ CSA at J1 equal to zero or negative & 22.7 & 77.5 & 68.4 & 31.8 \\
\hline
\end{tabular}


IJV and what as a pathological vein. For example, some researchers define $50 \%$ luminal restriction of the IJV as pathological [17], yet others argue that such a narrowing should be interpreted as abnormal only if accompanied by flow irregularities (for example, collateral outflow) irrespective of nominal degree of the stenosis [19, 22]. It is also important how such a narrowing is measured [23]. Other definitions of venographic anomaly in the territory of IJV are debatable as well [19]. Moreover, an angiographic catheter inserted in a retrograde manner can change the morphology of the jugular valve, producing false positive and negative results [19]. Therefore, it is possible that a number of abnormalities, especially valve lesions, were misdiagnosed in our patients.

There are also potential limitations of this study related both to sonographic and MR venographic assessment. The most important limitation of the sonographic part of our study is the fact that Doppler sonography is an operator-dependent test $[5,24,25]$. It is also known that CCSVI is more likely to be diagnosed using ultrasound in a mildly dehydrated patient (for example, after fasting overnight) [26] and that diagnosis of CCSVI given with the use of Doppler sonography is poorly reproducible [24]. Similarly, head rotation can significantly affect flow in the IJV, which may result in inadequate diagnosis of CCSVI [27]. The other weak point of our study is the fact that we did not assess intracranial veins (reflux in the intracranial veins is one of the original Zamboni's criteria). There is much controversy about studying sonographically cerebral veins. Most of the researchers agree that detection of a reflux in the deep cerebral veins is very difficult to perform and requires great expertise [28]. Yet, even if the assessment of intracranial veins were found highly sensitive and specific (which is probably not the case), considering the high rates of false positive findings of the remaining criteria (Table 1) it is unlikely that diagnostic accuracy of the whole set of five Zamboni's criteria would be greatly improved. The other questionable point of our study is our interpretation of all sonographically detectable valves as an abnormality. Unfortunately, published guidelines do not give a clear definition of such pathological intraluminal structures $[12,20]$. Undoubtedly, this part of sonographic assessment of IJVs needs clarification, and perhaps intravascular ultrasound (IVUS), instead of standard sonography, should be used to distinguish normal from pathological valves $[16,29,30]$.

The most obvious limitation of our MR assessment of the IJVs is the fact that probably we utilised a sub-optimal imaging protocol. Magnetic resonance venography is usually considered an objective and not operator-dependent diagnostic modality. With the use of MR venography the IJVs can be evaluated from their origins inside the skull (which cannot be done by means of ultrasound) to their junctions with the brachiocephalic veins. Theoretically, MR venography has the potential to replace other diagnostic methods. Moreover, in the case of evaluation of
MS patients this test can be combined with structural and functional imaging of the brain and cerebral vessels [20]. However, MR venography is actually not a single imaging technique, but rather a collection of very different diagnostic modalities. At the moment, no widely accepted and reliable MR protocol for the assessment of CCSVI exists [20]. In addition, published evidence is quite discordant. While some researchers who utilised MR venography in MS patients demonstrated obvious abnormalities of the veins [31-35], others were unable to demonstrate lesions $[36,37]$. Nevertheless, probably not all research utilised the correct imaging protocols. Of note, the study that used more sophisticated IJV-dedicated MR protocols revealed more stenoses in MS patients (55\% and 61\%) than in the control group (5\% and 10\%) [32].

Conventional MR venography that primarily focuses on the morphology of the vein can easily reveal a narrowing of its upper segment and also a flattening of its middle part. Yet pathological role of such narrowing of the upper and middle part of the IJV remains elusive [38]. Conversely, standard gadolinium-enhanced MR venography is not good in demonstrating the pathology of the jugular valves. Jugular valves are tiny structures, which are not well visible on standard MR images. Also, artefacts resulting from respiratory and cardiac movements routinely blur the area of these valves, and so does gadolinium contrast. Also, this imaging technique cannot reveal venous flow restrictions, which appear to be the most common CCSVI abnormality. But the pathology of jugular valves usually results in flow restriction that can be demonstrated by MR protocols focusing on flow assessment. The researchers who studied the flow characteristics in the IJVs in MS patients by means of 2-dimensional phase contrast MR imaging found that stenoses revealed by morphological MR venography significantly affected the flow. Patients diagnosed with stenoses showed significantly reduced jugular flow compared to non-stenotic patients. In addition, localisation of stenoses in the lower part of the IJV (the area of the jugular valve) led to more severe reduction of the flow. Interestingly, total arterial flow was not different in the patients with and without stenoses; therefore, probably cerebral venous outflow in 'stenotic' patients was shifted from the IJVs to an alternative venous network [39-41]. Recently, a sophisticated CCSVI-dedicated MR venography protocol has been recommended. This protocol, in addition to non-contrast venography based on the 2-dimensional time-of-flight imaging, utilises also the time-resolved, contrast-enhanced, 3-dimensional MR venography and the 3-dimensional, phase-contrast MR venography. The former contrast-enhanced imaging serves the purpose of evaluating vascular abnormalities, such as: vessel patency, stenoses, aplasia, truncular malformations, and valve abnormalities. The latter is used to assess flow dynamics in the veins and arteries of the neck [20]. However, as yet this promising protocol has not been tested against 
catheter venography or other invasive techniques, such as IVUS.

In our study we used CCSVI-dedicated MR imaging protocols that focused on the detection of venous outflow impairment. Since statistical coefficients characterising the diagnostic accuracy of our CCSVI-dedicated MR venography were found to be superior to those of Doppler sonography, it suggests that our approach was correct, but an optimisation of imaging protocols (such as modified: repetition time, echo time, acquisition time, or other imaging parameters) will be needed. Since CCSVI is primarily associated with compromised venous outflow from the central nervous system resulting in hypoperfusion of the brain, perhaps additional MR protocols aimed at perfusion assessment should also be used in the future.

\section{CONCLUSIONS}

Our research shows a clear gap in the understanding of venous haemodynamics in the territory of the IJVs, and more research is needed to improve the diagnostic accuracy of non-invasive tests. The results of our dedicated MR protocols were better when compared to Doppler sonography or traditional MR assessment utilising gadolinium-enhanced imaging. However, even these protocols were far from perfect, and it could be concluded that at the moment neither MR venography nor Doppler sonography should be used as a definitive test for diagnosing CCSVI.

\section{The authors declare no conflict of interest.}

\section{References}

1. Zamboni P., Galeotti R., Menegatti E., Malagoni A.M., Tacconi G., Dall'Ara S., Bartolomei I., Salvi F. Chronic cerebrospinal venous insufficiency in patients with multiple sclerosis. J Neurol Neurosurg Psychiatry 2009; 80: 392-399.

2. Zamboni P., Galeotti R. The chronic cerebrospinal venous insufficiency syndrome. Phlebology 2010; 25: 269-279.

3. Simka M. Chronic cerebrospinal venous insufficiency: current perspectives. J Vasc Diagn 2014; 2: 1-13.

4. Chambers B., Chambers J., Cameron H., Macdonell R. Chronic cerebrospinal venous insufficiency is not more prevalent in patients with mild multiple sclerosis: a sonographer-blinded, case-control ultrasound study. Mult Scler 2012; 19: 749-756.

5. Comi G., Battaglia M.A., Bertolotto A., Del Sette M., Ghezzi A., Malferrari G., Salvetti M., Sormani M.P., Tesio L., Stolz E., Zaratin P., Mancardi G. Observational case-control study of the prevalence of chronic cerebrospinal venous insufficiency in multiple sclerosis: results from the CoSMo study. Mult Scler 2013; 19: 1508-1517.

6. Leone M.A., Raymkulova O., Naldi P., Lochner P., Bolamperti L., Coppo L., Stecco A., Liboni W. Chronic cerebrospinal venous insufficiency is not associated with multiple sclerosis and its severity: a blind-verified study. PLoS One 2013; 8: e56031.

7. Zaniewski M., Kostecki J., Kuczmik W., Ziaja D., Opala G., Świat M., Korzeniowski T., Majewski E., Urbanek T., Pawlicki K.
Neck duplex Doppler ultrasound evaluation for assessing chronic cerebrospinal venous insufficiency in multiple sclerosis patients. Phlebology 2013; 28: 24-31.

8. Simka M., Ludyga T., Latacz P., Kazibudzki M. Diagnostic accuracy of current sonographic criteria for the detection of outflow abnormalities in the internal jugular veins. Phlebology 2013; 28: 285-292.

9. Ludyga T., Kazibudzki M., Simka M., Hartel M., Świerad M., Piegza J., Latacz P., Sedlak L., Tochowicz M. Endovascular treatment for chronic cerebrospinal venous insufficiency: is the procedure safe? Phlebology 2010; 25: 286-295.

10. Simka M., Latacz P., Ludyga T., Kazibudzki M., Świerad M., Janas P., Piegza J. Prevalence of extracranial venous abnormalities: results from a sample of 586 multiple sclerosis patients. Funct Neurol 2011; 26: 197-203.

11. Zamboni P., Menegatti E., Galeotti R., Malagoni A.M., Tacconi G., Dall'Ara S., Bartolomei I., Salvi F. The value of cerebral Doppler venous haemodynamics in the assessment of multiple sclerosis. J Neurol Sci 2009; 282: 21-27.

12. Nicolaides A.N., Morovic S., Menegatti E., Viseiner G., Zamboni P. Screening for chronic cerebrospinal venous insufficiency (CCSVI) using ultrasound. Recommendations for a protocol. Funct Neurol 2011; 26: 229-248.

13. Hartel M., Kluczewska E., Simka M., Ludyga T., Kostecki J., Zaniewski M. Magnetic resonance venography of chronic cerebrospinal venous insufficiency in patients with associated multiple sclerosis. Pol J Radiol 2011; 76: 59-62.

14. Denislic M., Milosevic Z., Zorc M., Ravnik I.Z., Mendiz O. Disability caused by multiple sclerosis is associated with the number of extracranial venous stenoses: possible improvement by venous angioplasty. Results of a prospective study. Phlebology 2013; 28: 353-360.

15. Petrov I., Grozdinski L., Kaninski G., Iliev N., Iloska M., Radev A. Safety profile of endovascular treatment for chronic cerebrospinal venous insufficiency in patients with multiple sclerosis. J Endovasc Ther 2011; 18: 314-323.

16. Scalise F., Farina M., Manfredi M., Auguadro C., Novelli E. Assessment of jugular endovascular malformations in chronic cerebrospinal venous insufficiency: colour-Doppler scanning and catheter venography compared with intravascular ultrasound. Phlebology 2013; 28: 409-417.

17. Traboulsee A.L., Knox K.B., Machan L., Zhao Y., Yee I., Rauscher A., Klass D., Szkup P., Otani R., Kopriva D., Lala S., Li D.K., Sadovnick D. Prevalence of extracranial venous narrowing on catheter venography in people with multiple sclerosis, their siblings and unrelated healthy controls: a blinded, case-control study. Lancet 2014; 383: 138-145.

18. Veroux P., Giaquinta A., Perricone D., Lupo L., Gentile F., Virgilio C., Carbonaro A., De Pasquale C., Veroux M. Internal jugular veins outflow in patients with multiple sclerosis: a catheter venography study. J Vasc Intervent Radiol 2013; 24: 1790-1797.

19. Simka M., Hubbard D., Siddiqui A.H., Dake M.D., Sclafani S.J., Al-Omari M., Eisele C.G., Haskal Z.J., Ludyga T., Miloševič Z.V., Sievert H., Stehling M.K., Zapf S., Zorc M. Catheter venography for the assessment of internal jugular veins and azygos vein: position statement by expert panel of the International Society for Neurovascular Disease. Vasa 2013; 42: 168-176.

20. Zivadinov R., Bastianello S., Dake M.D., Ferral H., Haacke E.M., Haskal Z.J., Hubbard D., Liasis N., Mandato K., Sclafani S., Siddiqui A.H., Simka M., Zamboni P. Recommendations for multimodal noninvasive and invasive screening for detection of extracranial venous abnormalities indicative of chronic cer- 
ebrospinal venous insufficiency: a position statement of the International Society for Neurovascular Disease. J Vasc Interv Radiol 2014; 25: 1785-1794.

21. Al-Omari M.H., Al-Bashir A. Internal jugular vein valve morphology in the patients with chronic cerebrospinal venous insufficiency (CCSVI); angiographic findings and schematic demonstrations. Rev Recent Clin Trials 2012; 7: 83-87.

22. Ferral H. Review of catheter venography protocols in the evaluation of chronic cerebrospinal venous insufficiency. Phlebol Rev 2014; 22: 11-17.

23. Zamboni P. How to objectively assess jugular primary venous obstruction. Veins Lymphatics 2014; 3: 74-76.

24. Leone M., Raymkulova O., Lucenti A., Stecco A., Bolamperti L., Coppo L., Liboni W., Rivadossi G., Zaccala G., Maggio M., Melis F., Giaccone C., Carriero A., Lochner P. A reliability study of color Doppler sonography for the diagnosis of chronic cerebrospinal venous insufficiency shows low inter-rater agreement. BMJ Open 2013; 3: e003508.

25. Menegatti E., Genova V., Tessari M., Malagoni A.M., Bartolomei I., Zuolo M., Galeotti R., Salvi F., Zamboni P. The reproducibility of colour Doppler in chronic cerebrospinal venous insufficiency associated with multiple sclerosis. Int Angiol 2010; 29: 121-126.

26. DiaconuC.I.,FoxR.J.,GrattanA.,Rae-GrantA.,LuM.,GornikH.L., Kim E.S. Hydration status substantially affects chronic cerebrospinal venous insufficiency assessment. Neurol Clin Pract 2013; 3: 386-391.

27. Farina M., Novelli E., Pagani R. Cross-sectional area variations of internal jugular veins during supine head rotation in multiple sclerosis patients with chronic cerebrospinal venous insufficiency: a prospective diagnostic controlled study with duplex ultrasound investigation. BMC Neurology 2013; 13: 162.

28. Tromba L., Blasi S., Vestri A., Kiltzanidi D., Tartaglia F., Redler A. Prevalence of chronic cerebrospinal venous insufficiency in multiple sclerosis: a blinded sonographic evaluation. Phlebology 2015; 30: 52-60.

29. Karmon Y., Zivadinov R., Weinstock-Guttman B., Marr K., Valnarov V., Dolic K., Kennedy C.L., Hojnacki D., Carl E.M., Hagemeier J., Hopkins L.N., Levy E.I., Siddiqui A.H. Comparison of intravascular ultrasound with conventional venography for detection of extracranial venous abnormalities indicative of chronic cerebrospinal venous insufficiency. J Vasc Interv Radiol 2013; 24: 1487-1498.

30. Lugli M., Morelli M., Guerzoni S., Maleti O. The hypothesis of patho-physiological correlation between chronic cerebrospinal venous insufficiency and multiple sclerosis: rationale of treatment. Phlebology 2012; 27 (Suppl 1): 178-186.

31. Dolic K., Marr K., Valnarov V., Dwyer M.G., Carl E., Karmon Y., Kennedy C., Brooks C., Kilanowski C., Hunt K., Siddiqui A.H., Hojnacki D., Weinstock-Guttman B., Zivadinov R. Intra- and extra luminal structural and functional venous anomalies in multiple sclerosis as evidenced by 2 noninvasive imaging techniques. AJNR Am J Neuroradiol 2012; 33: 16-23.

32. Rahman M.T., Sethi S.K., Utriainen D.T., Hewett J.J., Haacke E.M. A comparative study of magnetic resonance venography techniques for the evaluation of the internal jugular veins in multiple sclerosis patients. Magn Res Imaging 2013; 31: 1668-1676.

33. Zaharchuk G., Fischbein N.J., Rosenberg J., Herfkens R.J., Dake M.D. Comparison of MR and contrast venography of the cervical venous system in multiple sclerosis. Am J Neuroradiol 2011; 32: 1482-1489.
34. Zivadinov R., Karmon Y., Dolic K., Hagemeier J., Marr K., Valnarov V., Kennedy C.L., Hojnacki D., Carl E.M., Hopkins L.N., Levy E.I., Weinstock-Guttman B., Siddiqui A.H. Multimodal noninvasive and invasive imaging of extracranial venous abnormalities indicative of CCSVI: results of the PREMiSe pilot study. BMC Neurology 2013; 13: 151.

35. Zivadinov R., Lopez-Soriano A., Weinstock-Guttman B., Schirda C.V., Magnano C.R., Dolic K., Kennedy C.L., Brooks C.L., Reuther J.A., Hunt K., Andrews M., Dwyer M.G., Hojnacki D.W. Use of MR venography for characterization of the extracranial venous system in patients with multiple sclerosis and healthy control subjects. Radiology 2011; 258: 562-570.

36. Brod S.A., Kramer L.A., Cohen A.M., Barreto A.D., Bui T.T., Jemelka J.R., Ton K., Lindsey J.W., Nelson F., Narayana P.A., Wolinsky J.S. Chronic cerebrospinal venous insufficiency: masked multimodal imaging assessment. Mult Scler 2013; 19: 1499-1507.

37. Doepp F., Würfel J.T., Pfueller C.F., Valdueza J.M., Petersen D., Paul F., Schreiber S.J. Venous drainage in multiple sclerosis: a combined MRI and ultrasound study. Neurology 2011; 77: 1745-1751.

38. McTaggart R.A., Fischbein N.J., Elkins C.J., Hsiao A., Cutalo M.J., Rosenberg J., Dake M.D., Zaharchuk G. Extracranial venous drainage patterns in patients with multiple sclerosis and healthy controls. AJNR Am J Neuroradiol 2012; 33: 1615-1620.

39. Feng W., Utriainen D., Trifan G., Elias S., Sethi S., Hewett J., Haacke E.M. Characteristics of flow through the internal jugular veins and cervical C2/C3 and C5/C6 levels for multiple sclerosis patients using MR phase contrast imaging. Neurol Res 2012; 34: 802-809.

40. Feng W., Utriainen D., Trifan G., Sethi S., Hubbard D., Haacke E.M. Quantitative flow measurements in the internal jugular veins of multiple sclerosis patients using magnetic resonance imaging. Rev Rec Clin Trials 2012; 7: 117-126.

41. Haacke E.M., Feng W., Utriainen D., Trifan G., Wu Z., Latif Z., Katkuri Y., Hewett J., Hubbard D. Patients with multiple sclerosis with structural venous abnormalities on MR imaging exhibit an abnormal flow distribution of the internal jugular veins. J Vasc Interv Radiol 2012; 23: 60-68. 